Nutrition Science en évolution

La revue de l'Ordre professionnel des diététistes du Québec

\title{
Conférence annuelle de la Society for Nutrition Education and Behavior
}

\section{Caroline Langlais}

Volume 16, numéro 3, hiver 2019

URI : https://id.erudit.org/iderudit/1059222ar

DOI : https://doi.org/10.7202/1059222ar

Aller au sommaire du numéro

Éditeur(s)

Ordre professionnel des diététistes du Québec

ISSN

2561-620X (numérique)

Découvrir la revue

Citer ce document

Langlais, C. (2019). Conférence annuelle de la Society for Nutrition Education and Behavior. Nutrition Science en évolution, 16(3), 36-38.

https://doi.org/10.7202/1059222ar d'utilisation que vous pouvez consulter en ligne. 

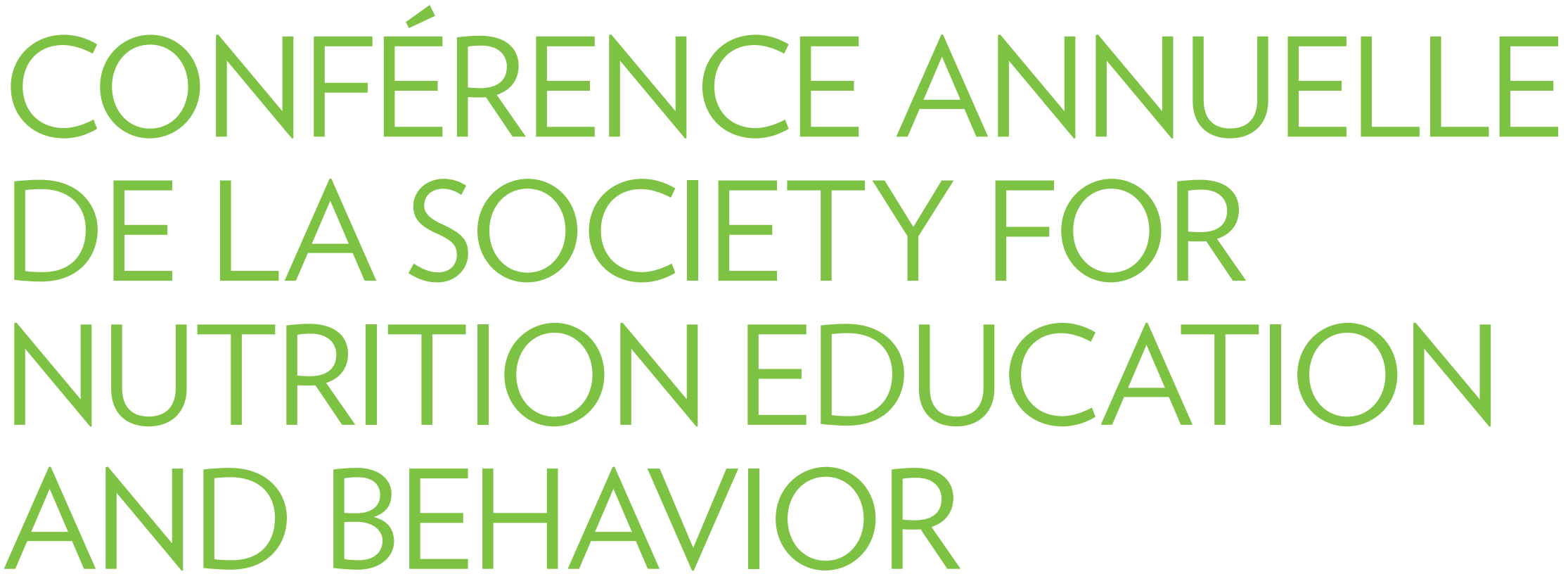

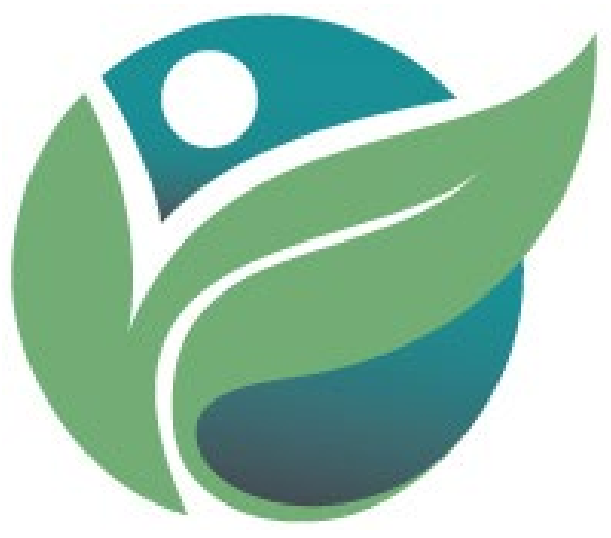

\section{ant \\ VIABILITÉ DU SYSTĖME ALIMENTAIRE : SCIENCE ET BESOINS FUTURS DE RECHERCHE} la $51^{e}$ conférence annuelle de la Society for Nutrition Education and Behavior (SNEB) à Minneapolis, au Minnesota. L'alimentation durable était au cour de ce congrès dont le thème était Optimizing impact Supporting Food Systems with Nutrition Education.

Trois conférences sont ici résumées.

\section{Michael W. Hamm,} professeur d'agriculture durable et chercheur principal au Center for Regional Food Systems, Department of Community Sustainability, Université du Michigan

Michael W. Hamm a expliqué d'entrée de jeu que la planète a des limites, lesquelles définissent la marge de manœuvre sécuritaire avec laquelle l'humain doit composer ${ }^{[1]}$. Il avance que les limites ont été dépassées, témoin les changements climatiques, mais aussi la perte de biodiversité (terrestre et marine) et l'ingérence de l'humain dans le cycle de l'azote. On ne devrait donc pas seulement s'inquiéter des émissions de car- bone, mais aussi de la manière dont les terres et les ressources océaniques sont utilisées. À son avis, il ne fait aucun doute que l'agriculture moderne est une cause majeure de pollution environnementale.

Pour répondre aux besoins alimentaires mondiaux, il faudra, selon ce professeur, mettre en place simultanément les quatre approches suivantes $^{[2]}$ :

$>$ Une modification des habitudes alimentaires ;

$>$ L'adoption de pratiques de production agricole qui préservent les ressources;

> Une répartition plus équitable des ressources;

$>$ La réduction du gaspillage alimentaire.

Selon M. Hamm, pour y parvenir, nous devrons complètement changer notre alimentation (p. ex. réduire la surconsommation, diminuer la consom- 
mation de viande en intégrant les insectes à notre alimentation comme source de protéines).

Il deviendra primordial d'utiliser les terres de manière à maximiser le nombre de personnes nourries. Plusieurs autres variables sont à considérer selon le conférencier comme, entre autres, l'augmentation constante de la population, la localisation de la production (transport, besoins en chauffage ou en électricité dans les serres, besoins en eau plus grands dans certaines régions).

M. Hamm a conclu sa présentation en soulignant que le rapport du Dietary Guidelines Advisory Committee (DGAC) des États-Unis émet des recommandations officielles sur l'alimentation durable ${ }^{[3]}$. Ces recommandations sont prises en considération par le U.S. Department of Agriculture (USDA) et le US Departement of Health and Human Services (HHS) pour émettre les recommandations alimentaires officielles destinées aux Américains (Dietary Guidelines for Americans):

1. Mener des études pour déterminer si les régimes alimentaires durables sont abordables et accessibles à tous ;

2. Concevoir et mener des analyses approfondies des habitudes alimentaires des Américains. Le but? Déterminer dans quelle mesure l'alimentation durable est un aspect important lors de leurs choix alimentaires et comment les sensibiliser à ce sujet;

3. Comprendre la manière dont les pratiques de production, les décisions en matière de chaîne d'approvisionnement, les comportements des consommateurs et l'élimination des déchets affectent la viabilité de l'environnement;

4. Déterminer les bénéfices économiques potentiels et les défis auxquels les différents acteurs de la chaîne d'approvisionnement peuvent s'attendre.
LES ENJEUX POLITIQUES LIÉS À L'ALIMENTATION DURABLE

\section{Kate Clancy,}

consultante en systèmes

alimentaires, Briggs Nutrition

Science Symposium

Selon Kate Clancy, une alimentation durable doit tenir compte des six facteurs ${ }^{[4]}$ suivants tous aussi importants les uns que les autres:

1. Besoins en aliments et en nutriments, sécurité alimentaire et accessibilité;

2. Bien-être et santé;

3. Biodiversité, environnement, climat;

4. Héritage culturel et habiletés;

5. Produits écoresponsables, alimentation locale, aliments de saison;

6. Commerce équitable.

À son avis, des politiques alimentaires mieux intégrées, englobant tous ces facteurs, sont essentielles pour la stabilité mondiale et la sécurité alimentaire ${ }^{[5]}$.

La consultante a enchaîné en présentant quelques caractéristiques importantes d'une alimentation durable compatible avec la santé, selon la FAO (Food and Agriculture Organization ${ }^{[6]}$ :

$>$ La consommation d'une grande variété d'aliments ;

$>$ Un bon équilibre entre les apports énergétiques et les besoins individuels (éviter la surconsommation);

$>$ Une alimentation basée sur la consommation de fruits, de légumes, de légumineuses et de grains entiers et la modération de celle de la viande.

Toutefois, elle avance qu'il est difficile de transmettre des recommandations relativement à l'alimentation durable. En voici deux raisons :

$>$ Les professionnels de la santé n'ont pas nécessairement une bonne compréhension des effets de l'alimentation durable.
Ils peuvent ne pas avoir une vue d'ensemble des effets environnementaux, sociaux et économiques de la production et de la consommation;

$>$ L'industrie alimentaire et le gouvernement ne concordent pas toujours sur le sujet.

Au dire de Kate Clancy, voici les actions qui devraient être mises en place prochainement:

$>$ Améliorer la formation des diététistes/nutritionnistes quant à l'alimentation durable et aux systèmes alimentaires impliqués ;

$>$ Continuer à investir dans la recherche sur l'alimentation durable;

$>$ Fournir un cadre politique rationnel pour guider les producteurs et les consommateurs vers l'agriculture et l'alimentation durables ;

$>$ Étudier les efforts faits par d'autres pays relativement à l'alimentation durable;

> Travailler à l'échelle nationale, régionale et locale pour accroître le débat et l'action concernant l'alimentation durable.

\section{TRADUIRE LA SCIENCE DE L'ALIMENTATION DURABLE: COMMENT L'ÉDUCATION NUTRITIONNELLE PEUT-ELLE ÊTRE UTILE?}

\section{Mark David Richards,}

vice-président directeur, gestion, KRC Research

Selon Monsieur Richards, il peut être difficile pour un consommateur de bien comprendre les enjeux environnementaux pour plusieurs raisons :

$>$ La surabondance de l'information;

$>$ La multiplication et l'évolution rapide des données scientifiques et des découvertes;

$>$ La nature parfois contradictoire des informations qui circulent; 
$>$ Les changements relatifs à la culture, aux croyances et aux normes qu'ils supposent;

$>$ Le faible niveau de littératie scientifique en général;

$>$ Un certain scepticisme et une perte de confiance envers les institutions et les dirigeants.

Malgré cela, le concept d'alimentation durable est perçu de manière très favorable par les Américains ${ }^{[7]}$. Dans un sondage réalisé en 2018, auquel renvoie M. Richards, $59 \%$ des gens affirment qu'il est important que les produits alimentaires qu'ils achètent ou consomment soient produits de manière durable. Toutefois, l'alimentation durable semble un concept nébuleux pour beaucoup de consommateurs. En effet, $24 \%$ des Américains sondés avouent ne pas savoir si le système alimentaire américain est durable ${ }^{[8]}$.

Voici quelques suggestions de M. Richards pour communiquer efficacement des messages relatifs à l'alimentation durable:
1. Être à l'écoute de son auditoire. Sont-ils en désaccord avec les experts? Quelles sont leurs priorités et leurs préoccupations? Que veulent-ils savoir et qu'ils ne vous disent pas?

2. Simplifier et affiner le message. Éviter le jargon; dresser un portrait clair; établir un lien de confiance en privilégiant la compassion plutôt que les statistiques.

3. Utiliser des messagers en lesquels les gens ont confiance. Les gens croient les personnes qui partagent leurs valeurs et auxquelles ils s'identifient. Engager donc des communicateurs créatifs qui toucheront le cœur et l'esprit des gens.

4. Adapter son message à la sensibilité du public. Il est rare de pouvoir rejoindre tout le monde. Aussi, vaut-il mieux se concentrer sur ceux qui démontrent de l'intérêt. Des communications numériques ciblées, entre autres, peuvent donner des résultats intéressants.

\section{Références}

5. Rockstrom, J., Steffen, W., Noone, K., Persson, A., Chapin, F. S., 3rd, Lambin, E. F., Foley, J. A. (2009a). A safe operating space for humanity. Nature, 461(7263), 472-475.

6. Garnett T. Food sustainability: problems, perspectives and solutions. Proc Nutr Soc. 2013;72(1):29-39.

7. https://health.gov/dietaryguidelines/2015-scientific-report/pdfs/scientific-report-of-the-2015-dietary-guidelines-advisory-committee.pdf.

8. Mason, P., and Lang, T. 2017. Sustainable Diets. Routledge. London and New York. 354 pages.

9. Candel JL, Pereira, L. Towards integrated food policy: Main challenges and steps ahead. Environmental Science \& Policy. Vol 73, July 2017;73:89-92.

10. Fischer, C.G., Garnett, T. 2016. Plates, pyramids, planet. Developments in national healthy and sustainable dietary guidelines: a state of play assessment. Food and Agriculture Organization of the United Nations and The Food Climate Research Network at the University of Oxford.

11. International Food Information Council (IFIC) Foundation, survey of 1009 U.S. adults 18-80, March 2018.

12. KRC Research, survey of 1,206 U.S. adults conducted July 19-20, 2018.

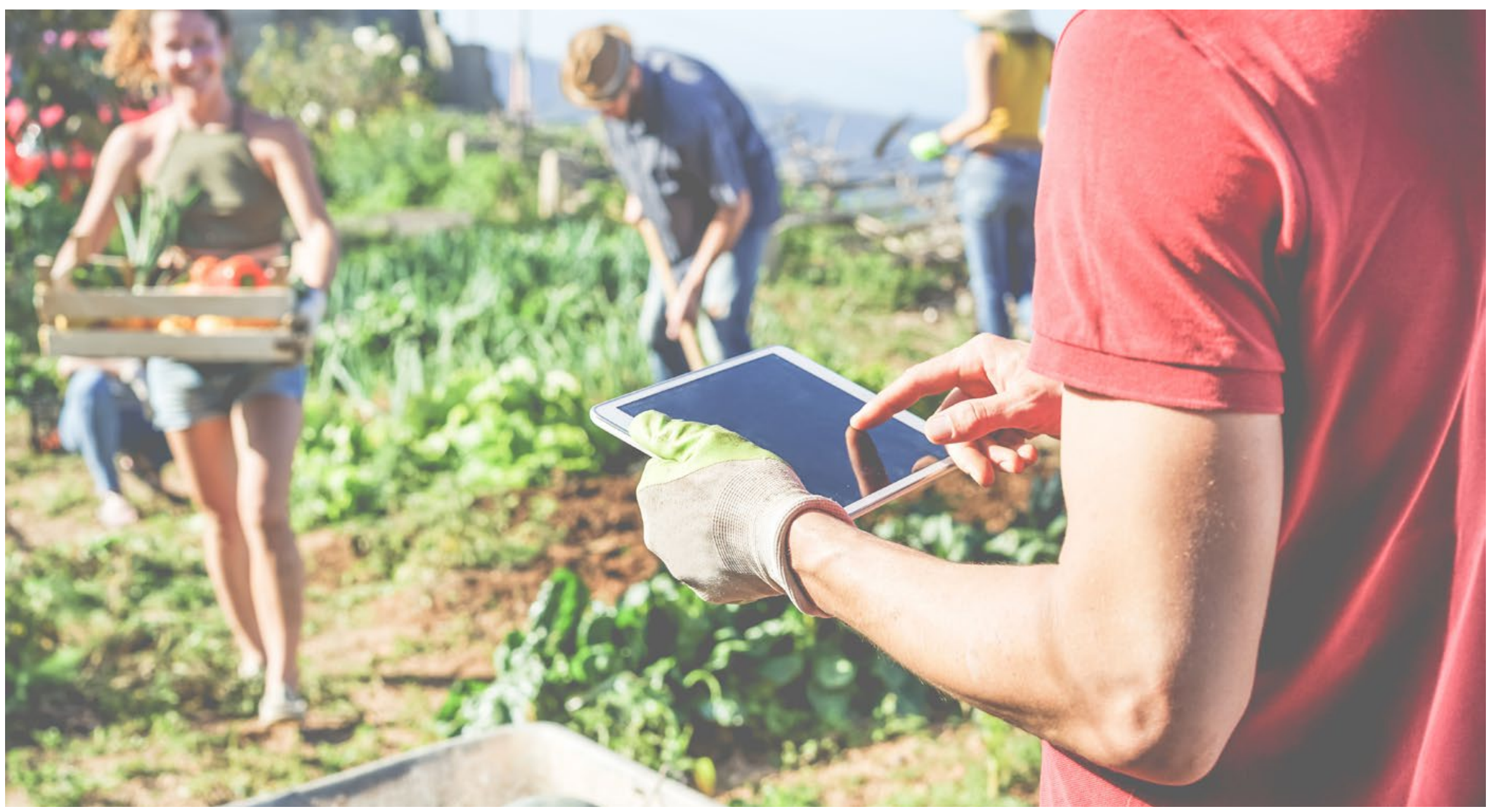

